\title{
ON THE SPECTRUM OF THE WANNIER-STARK OPERATOR
}

\author{
A. A. POZHARSKII
}

\section{BASIC RESULTS}

We consider the one-dimensional Schrödinger equation

$$
-\psi^{\prime \prime}-F x \psi+p(x) \psi=E \psi
$$

on the semiaxis $0 \leq x<+\infty$. Here the potential $p(x)$ is a periodic real function, $p(x+1)=p(x)$, satisfying the condition $p(x) \in L_{1}[0,1]$. We assume that the constant $F$ is positive and that $\int_{0}^{1} p(x) d x=0$; the latter condition can always be ensured by a shift of the spectral parameter $E$.

We introduce the operator

$$
H=-\frac{d^{2}}{d x^{2}}-F x+p(x)
$$

in $L_{2}\left(\mathbb{R}_{+}\right)$with the domain

$$
\operatorname{dom}(H)=\left\{\varphi: \begin{array}{l}
\varphi^{\prime} \text { is absolutely continuous, } \varphi(0)=0, \operatorname{supp} \varphi \text { is } \\
\text { bounded, and }-\varphi^{\prime \prime}+p \varphi \in L_{2}\left(\mathbb{R}_{+}\right)
\end{array}\right\} .
$$

Remark. The function $-\varphi^{\prime \prime}+p(x) \varphi$ belongs to $L_{2}\left(\mathbb{R}_{+}\right)$if $\varphi \in \operatorname{dom}(H)$, but $\varphi^{\prime \prime}$ and $p \varphi$ separately may fail to belong to $L_{2}\left(\mathbb{R}_{+}\right)$. However, they belong to $L_{1}\left(\mathbb{R}_{+}\right)$.

The operator $H$ is essentially selfadjoint. This fact is proved in $\S 5$. We denote by $H_{d}$ the closure of $H$.

In the case where the potential $p(x)$ is smooth, equation (1) and the operator $H_{d}$ have been studied thoroughly; see $\mathrm{AGZ}, \mathrm{AZ}, \mathrm{Ba}, \mathrm{B2}, \mathrm{BD1}, \mathrm{BD2}, \mathrm{NN}$. In the case of a nonsmooth potential $p(x)$, only few results are known (see [B1, [DSS, [E, $[\mathrm{P}$ ).

We introduce the notation

$$
r(l)=e^{-i \frac{3 \pi}{4}} \frac{1}{\sqrt{2 F}} \int_{0}^{1} p(t) e^{-2 i \pi l t} d t, \quad \omega(l)=\int_{0}^{1} \int_{0}^{t} p(y) p(t) e^{2 i \pi l t} d y d t .
$$

In $[\mathrm{P}$, the following Theorem 1 was proved.

Assumption (A). $\sum_{l=1}^{\infty}|r(l)| l^{-\frac{1}{2}}<\infty, \sum_{l=1}^{\infty}|\omega(l)| l^{-1}<\infty$.

Assumption (B). $\sum_{l=1}^{\infty}|r(l)|^{2} l^{-\frac{1}{2}}<\infty, \sum_{l=1}^{\infty}|r(l)| l^{-\frac{3}{4}}<\infty, \sum_{l=1}^{\infty}|\omega(l)| l^{-1}<\infty$.

Theorem 1. i) If the potential $p(x)$ satisfies Assumption (A), then the spectrum of the operator $H_{d}$ is absolutely continuous and fills the real axis.

ii) If the potential $p(x)$ satisfies Assumption (B), then the absolutely continuous spectrum of $H_{d}$ fills the real axis.

In the present paper, we study the absolutely continuous spectrum of $H_{d}$ and generalize the results of Theorem 1. Our main result in this paper is Theorem 2 below.

2000 Mathematics Subject Classification. Primary 34L40.

Key words and phrases. Schrödinger operator, essentially selfadjoint operator, continuous spectrum. 
Assumption (C). $\sum_{l=1}^{\infty}|r(l)| l^{-1}<\infty, \sum_{l=1}^{\infty}|\omega(l)| l^{-1}<\infty$.

Remark. If on the interval $[0,1]$ the potential $p(x)$ is given by the equation $|p(x)|=$ $C_{1}\left|x-x_{0}\right|^{-1+\varepsilon}$ for $\varepsilon>0$, then Assumption (C) is valid.

Theorem 2. If $p(x)$ satisfies Assumption (C), then the absolutely continuous spectrum of $H_{d}$ fills the real axis.

The proof of this theorem is based on the results obtained in $[\mathrm{P}$. We state two results of [P] that we need in the sequel (Theorems 3 and 4).

Theorem 3. Let $p(x)$ be a periodic potential absolutely integrable on the period and satisfying $\int_{0}^{1} p(t) d t=0$. Suppose $F>0$ and $E \in \mathbb{R}$. Then an arbitrary solution of equation (1) has the following asymptotic expansion as $x \rightarrow+\infty$ :

$$
\psi(x)=\frac{s_{l}}{\sqrt[4]{F x}} e^{i \frac{2}{3 F}(E+F x)^{3 / 2}}+\frac{t_{l}}{\sqrt[4]{F x}} e^{-i \frac{2}{3 F}(E+F x)^{3 / 2}}+O\left(\frac{\left\|\mathbf{s}_{l}\right\|}{\sqrt{x}}\right), \quad x \in I_{l} .
$$

Here $I_{l}=\left(\tilde{n}_{l-1}, \tilde{n}_{l}\right) \backslash\left(\tilde{i}_{l-1} \cup \tilde{i}_{l}\right), \tilde{n}_{l}=\left((\pi l)^{2}-E\right) / F$, and $\tilde{i}_{l}=\left\{n:\left|n-\tilde{n}_{l}\right|=O\left(l^{2 / 3}\right)\right\}$. On the adjacent intervals $I_{l}$ and $I_{l+1}$, the coefficients $s_{l}, t_{l}$ and $s_{l+1}, t_{l+1}$ are related by the transformation

$$
\mathbf{t}_{l+1}=\widetilde{W}_{l} \mathbf{t}_{l}, \quad \mathbf{t}_{l}=\left(\begin{array}{c}
s_{l} \\
t_{l}
\end{array}\right), \quad \widetilde{W}_{l}=\widetilde{W}_{l}(E),
$$

where

$$
\widetilde{W}_{l}=e^{-i \pi l \frac{E}{F} \sigma_{3}} S_{l} e^{i \pi l \frac{E}{F} \sigma_{3}}, \quad \sigma_{1} \bar{S}_{l} \sigma_{1}=S_{l}, \quad S_{l}=S_{l}(E) .
$$

The Pauli matrices $\sigma_{1}$ and $\sigma_{3}$ are given by $\sigma_{1}=\left(\begin{array}{ll}0 & 1 \\ 1 & 0\end{array}\right)$ and $\sigma_{3}=\left(\begin{array}{cc}1 & 0 \\ 0 & -1\end{array}\right)$. For large $l$, the matrix $S_{l}$ has the asymptotics

$$
S_{l}=\left(\begin{array}{cc}
1+q(l) l^{-1}+O\left(l^{-7 / 6}\right) & r(l) l^{-1 / 2} e^{\frac{2 i}{3 F}(\pi l)^{3}}+O\left(l^{-1} d(l)\right) \\
\bar{r}(l) l^{-1 / 2} e^{-\frac{2 i}{3 F}(\pi l)^{3}}+O\left(l^{-1} d(l)\right) & 1+q(l) l^{-1}+O\left(l^{-7 / 6}\right)
\end{array}\right),
$$

where

$$
q(l)=\frac{1}{2}|r(l)|^{2}, \quad d(l)=|r(l)|+|\omega(l)|+O\left(l^{-1 / 3}\right),
$$

and $r$ and $\omega$ are the functions defined in (2).

Furthermore, we have

$$
\int_{\tilde{n}_{l}}^{\tilde{n}_{l+1}}|\psi(x)|^{2} d x=\frac{2 \pi}{F}\left\|\mathbf{t}_{l}\right\|^{2}(1+o(1)) .
$$

We note that, for the first time, a similar result was proved by Buslaev in [B1 for the equation

$$
-\psi^{\prime \prime}-F x \psi+V \sum_{n=-\infty}^{+\infty} \delta(x-n) \psi=E \psi .
$$

Theorem 3 is a generalization of the results of [B1], and its proof is based on the ideas developed in B1].

Definition 1. A solution $\psi^{u}(x)$ of equation (1) is said to be subordinate at $+\infty$ if for each solution $\psi(x)$ linearly independent of $\psi^{u}(x)$ we have

$$
\lim _{x \rightarrow+\infty}\left(\int_{0}^{x}\left|\psi^{u}(t)\right|^{2} d t / \int_{0}^{x}|\psi(t)|^{2} d t\right)=0
$$


Definition 2. A solution $\mathbf{t}_{l}^{u}$ of system (3) is said to be subordinate if for each solution $\mathbf{t}_{l}$ linearly independent of $\mathbf{t}_{l}^{u}$ we have

$$
\lim _{L \rightarrow \infty}\left(\sum_{l=1}^{L}\left\|\mathbf{t}_{l}^{u}\right\|^{2} / \sum_{l=1}^{L}\left\|\mathbf{t}_{l}\right\|^{2}\right)=0 .
$$

Theorem 4. Suppose the assumptions of Theorem 3 are satisfied. Then equation (1) has a subordinate solution if and only if this is true for system (3).

Now, we state yet another result to be used in the sequel. The following theorem was proved in GP.

Theorem 5. If equation (1) has no subordinate solutions for almost any $E \in \mathbb{R}$ with respect to Lebesgue measure, then the absolutely continuous spectrum of $H_{d}$ fills the real axis.

The change of variables $\mathbf{s}_{l}=\exp \left(i \pi l \frac{E}{F} \sigma_{3}\right) \mathbf{t}_{l}$ reduces (3) to the system

$$
\mathbf{s}_{l+1}=e^{i \pi \frac{E}{F} \sigma_{3}} S_{l} \mathbf{s}_{l} .
$$

Moreover,

$$
\left\|\mathbf{s}_{l}\right\|=\left\|\mathbf{t}_{l}\right\| .
$$

In what follows, we study system (5) rather than (3).

Lemma 1. Suppose that the conditions of Theorem 3 are fulfilled, and that, for almost all $E \in \mathbb{R}$ with respect to Lebesgue measure, system (5) has no subordinate solutions. Then the absolutely continuous spectrum of $H_{d}$ fills the real axis.

Proof. By (6), systems (3) and (5) admit subordinate solutions simultaneously. Consequently, for almost all $E \in \mathbb{R}$ with respect to Lebesgue measure, system (3) has no subordinate solutions. Now, the claim follows from Theorems 4 and 5 .

Thus, the proof of Theorem 2 reduces to the study of asymptotic properties of system (5). Observe that this system is similar to the Schrödinger equation with slowly decaying potential (the matrix $S_{l}$ tends to the identity matrix as $l \rightarrow \infty$ ). Recently, for the Schrödinger operator with slowly decaying potential, a simple method of localization of the absolutely continuous spectrum was proposed in [DK]. This method is based on the use of certain spectral identities, which have recently received the name of BFZ (BuslaevFaddeev-Zakharov) identities; see $\overline{\mathrm{BF}}$. In the case of system (5), we follow the same idea. Some difficulties in the study of system (5) are due to the fact that the matrix $S_{l}$ may depend on the spectral parameter $E$. It is convenient to begin with a simpler system. Consider the two-dimensional recurrence system

$$
\mathbf{s}_{l+1}=z^{\sigma_{3}} W_{l} \mathbf{s}_{l}, \quad l=0,1, \ldots .
$$

Here $\mathbf{s}_{l} \in \mathbb{C}^{2}, z \in \mathbb{C} \backslash\{0\}$, and the $W_{l}$ are complex matrices of size $2 \times 2$ that satisfy the conditions

$$
\sigma_{1} \overline{W_{l}} \sigma_{1}=W_{l}, \quad \operatorname{det} W_{l}=1
$$

and do not depend on $z$. Conditions (8) imply that the matrices $W_{l}$ can be represented in the form

$$
W_{l}=\left(\begin{array}{cc}
w_{l} & v_{l} \\
\bar{v}_{l} & \bar{w}_{l}
\end{array}\right), \quad\left|w_{l}\right|^{2}-\left|v_{l}\right|^{2}=1 .
$$

The following theorem is the main result concerning system (7) (we note that the matrices $S_{l}$ may depend on the spectral parameter $E$, while the matrices $W_{l}$ are independent of $z$ ). 
Theorem 6. Suppose the matrices $W_{l}$ are representable in the form (9). We assume that the series

$$
\sum_{l=0}^{\infty}\left|v_{l}\right|^{2}
$$

converges and the limit

$$
\lim _{l \rightarrow \infty} W_{l}=I, \quad I=\left(\begin{array}{ll}
1 & 0 \\
0 & 1
\end{array}\right),
$$

exists. Then, for almost all $\phi \in[0,2 \pi)$ with respect to Lebesgue measure, system (7) has no subordinate solutions for $z=e^{i \phi}$.

Remark. We note that condition (11) can be lifted. To verify this, it suffices to make the change of variables $\mathbf{s}_{l}=e^{i \sum_{k=0}^{l-1} \arg w_{l} \sigma_{3}} \mathbf{t}_{l}$ in system (7).

The proof of Theorem 6 is based on the study of the Weyl function $m(z)$ (the definition of it is given in Theorem 7). We consider a specific basis of solutions of (7). Let $\theta(z)$ and $\varphi(z)$ be solutions of $(7)$ such that $\theta_{0}(z)=\left(\begin{array}{l}1 \\ 0\end{array}\right)$ and $\varphi_{0}(z)=\left(\begin{array}{l}0 \\ 1\end{array}\right)$. In what follows, by a solution $\theta(z)$ of system $(7)$ we mean a sequence $\left\{\theta_{l}(z)\right\}_{l=0}^{\infty}$ satisfying $(7)$.

Theorem 7. If the matrices $W_{l}$ are representable in the form (9), then there exists a Weyl function $m(z)$ analytic in $|z|<1$ and such that

$$
f(z)=\theta(z)+m(z) \varphi(z) \in l^{2}\left(\mathbb{Z}_{+}, \mathbb{C}^{2}\right), \quad 0<|z|<1 .
$$

Furthermore, $|m(z)|<1$ for $|z|<1$.

Theorem 7 is proved in $\S 1$.

Corollary 1. The Weyl function $m(z)$ has radial boundary value 11 $[0,2 \pi)$, almost everywhere with respect to Lebesgue measure.

Proof. The inequality $|m(z)|<1$ for $|z|<1$ shows that $m(z)$ belongs to the Hardy class $H_{\infty}(|z| \leq 1)$. This implies the required statement (see [Z] ).

The following lemma (to be proved in $\S 2$ ), plays a key role in the proof of Theorem 6 .

Lemma 2. If the series (10) converges, then

$$
-\int_{0}^{2 \pi} \ln \left(1-\left|m\left(e^{i \phi}\right)\right|^{2}\right) d \phi \leq 4 \pi \sum_{l=0}^{\infty} \ln \left|w_{l}\right| .
$$

Remark. The series $\sum_{l=0}^{\infty} \ln \left|w_{l}\right|$ and $\sum_{l=0}^{\infty}\left|v_{l}\right|^{2}$ converge simultaneously.

By this lemma, the Weyl function satisfies the estimate

$$
\left|m\left(e^{i \phi}\right)\right|<1
$$

for almost all $\phi$. Estimate (12) implies that system (7) has no subordinate solutions at $\phi$ (this fact can be proved by the methods of [GP]), and now the statement of Theorem 6 follows directly. However, the proof of this fact is relatively long, and we prove a simpler but slightly weaker statement. More precisely, we prove Theorem 6 for a set of $\phi$ that is narrower than the set of all $\phi$ for which estimate (12) is valid, but, certainly, is a set of full measure.

Theorem 6 follows easily from the next two lemmas. The main ideas of their proofs are borrowed from [LS]. We note that the proof of Lemma 3 is based on the results of Lemma 2. The proofs of Lemmas 3 and 4 are given in $\S 3$.

\footnotetext{
${ }^{1}$ The radial boundary values are defined by $m\left(e^{i \phi}\right)=\lim _{r \rightarrow 1-0} m\left(r e^{i \phi}\right)$.
} 
We represent system (7) in the matrix form:

$$
F_{l+1}=e^{i \phi \sigma_{3}} W_{l} F_{l}, \quad F_{0}=I .
$$

Lemma 3. Suppose the series (10) converges and

$$
\left\|W_{l}-I\right\| \leq 1 / 8, \quad l=0,1, \ldots .
$$

Let $a_{l}$ be a sequence of nonnegative numbers such that the series $\sum_{l \geq 0} a_{l}$ is convergent. Then, for almost all $\phi \in[0,2 \pi)$ with respect to Lebesgue measure, there exists a constant $C_{9}(\phi)<\infty$ such that

$$
\liminf _{L \rightarrow \infty} \frac{1}{L} \sum_{l=0}^{L}\left\|F_{l}\right\|^{2} \leq C_{9}(\phi), \quad \sum_{l=0}^{\infty} a_{l}\left\|F_{l}\right\|\left\|F_{l+1}\right\| \leq C_{9}(\phi) .
$$

Lemma 4. Let $B_{l}$ be a matrix solution of the system

$$
B_{l+1}=K_{l} B_{l}, \quad B_{0}=I,
$$

where the matrix $K_{l}$ satisfies $\operatorname{det} K_{l}=1$. If

$$
\liminf _{L \rightarrow \infty} \frac{1}{L} \sum_{l=0}^{L}\left\|B_{l}\right\|^{2}<\infty,
$$

then system (14) has no subordinate solutions.

We note that the matrices $K_{l}$ may depend on the spectral parameter.

Now we show how Theorem 6 can be derived from Lemmas 3 and 4 . Since the series (10) converges, there is a constant $N$ such that condition (13) is fulfilled for all $l \geq N$. Consider the auxiliary system obtained from the given system by replacing the first $N$ matrices $W_{l}$ by the identity matrix. Obviously, the behavior of a subordinate solution does not depend on the behavior of the matrices $W_{l}$ for finite $l$ (e.g., for $l \leq N$ ); therefore, the two systems in question have subordinate solutions simultaneously. We apply Lemma 3 and then Lemma 4 to the auxiliary system, putting $K_{l}=e^{i \phi \sigma_{3}} W_{l}$. As a result, we obtain the required statement.

Now, we proceed to system (5). The method used in the proof of Theorem 6 cannot be applied to system (5) because, in general, the matrices $S_{l}$ may depend on the spectral parameter $E$. Therefore, we are forced to perform the construction in two steps. For this, we represent the matrices $S_{l}$ in the form $S_{l}=W_{l}+V_{l}$, where the matrices $W_{l}$ satisfy the conditions of Theorem 6 and, in particular, do not depend on the spectral parameter $E$, and the matrices $V_{l}$ decay sufficiently fast (more precisely, we require the convergence of the series $\left.\sum_{l \geq 0}\left\|V_{l}\right\|\right)$, but may depend on $E$. From Theorem 6 it follows that the unperturbed system

$$
\mathbf{s}_{l}=e^{i \pi \frac{E}{F} \sigma_{3}} W_{l} \mathbf{s}_{l}
$$

has no subordinate solutions for almost all $E \in \mathbb{R}$.

Next, using the fact that the perturbation $V_{l}$ is small, we can compare the solutions of systems (5) and (15), thus arriving at Theorem 2. More precisely, we prove that, for almost all $\phi$, the solutions of systems (5) and (15) are related by a bounded matrix transformation $U_{l}$ (see Theorem 9). In $\S 4$, we give a rigorous proof of Theorem 2. Now, we outline the proof. We use the following notation: $F_{l}$ is a matrix solution of the unperturbed system (15), $B_{l}$ is a matrix solution of the perturbed system (5) with the matrices $S_{l}=W_{l}+V_{l}$, and the matrix solutions $B_{l}$ and $F_{l}$ are related to each other by 
the matrix transformation $U_{l}$ :

$$
\begin{aligned}
& F_{l+1}=e^{i \phi \sigma_{3}} W_{l} F_{l} \rightarrow \text { the Weyl function } m(z) \text { (Theorem 7) } \\
& \left.\rightarrow\left|m\left(e^{i \varphi}\right)\right|<1 \text { for a.e. } \varphi \text { (Lemma } 2\right) \rightarrow \text { Lemma } 3 \\
& B_{l+1}=K_{l} B_{l} \longrightarrow \text { Lemma } 4 \\
& F_{l+1}=e^{i \phi \sigma_{3}} W_{l} F_{l} \\
& \sum_{l \geq 0}\left|v_{l}\right|^{2}<\infty\left\{\begin{array}{lc}
\text { Lemma } 3 & \sum_{l \geq 0}\left|v_{l}\right|^{2}<\infty, \\
\sum_{l \geq 0}\left\|V_{l}\right\|<\infty
\end{array} \mid\right. \text { Lemma 3 } \\
& \liminf _{L \rightarrow \infty} L^{-1} \sum_{l=0}^{L}\left\|F_{l}\right\|^{2} \leq C(\phi)<\infty \\
& \text { for a.e. } \phi \in[0,2 \pi) \\
& \sum_{l=0}^{\infty}\left\|V_{l}\right\|\left\|F_{l}\right\|\left\|F_{l+1}\right\| \leq C(\phi)<\infty \\
& \text { for a.e. } \phi \in[0,2 \pi) \\
& \downarrow \text { see Theorem } 9 \\
& B_{l}=F_{l} U_{l},\left\|U_{l}\right\| \leq C(\phi)<\infty \\
& \liminf _{L \rightarrow \infty} L^{-1} \sum_{l=0}^{L}\left\|B_{l}\right\|^{2} \leq C(\phi)<\infty \text { for a.e. } \phi \in[0,2 \pi) \\
& \downarrow \text { Lemma } 4
\end{aligned}
$$

We note that the basic constructions are taken from the corresponding parts of the theory of the one-dimensional Schrödinger equation

$$
-\psi^{\prime \prime}+q(x) \psi=E \psi .
$$

For basic notions concerning the Weyl function for (16), see $\mathrm{L}$. The link between the boundary values of the Weyl function and the existence of a subordinate solution of (16) was established in GP. The idea to use the BFZ formulas in the study of the spectral properties of (16) is borrowed from [DK]. In the proofs of Lemmas 3 and 4, we used some ideas from [LS].

The present paper consists of five sections. In $\S 1$, we prove Theorem $7 ; \S 2$ contains the proof of Lemma 2. Lemmas 3 and 4 are proved in $\S 3$, and in $\S 4$ we prove Theorem 2 . Finally, in $\S 5$, we prove that the operator $H$ is essentially selfadjoint.

Acknowledgements. The author wants to thank Buslaev for numerous discussions and information about the literature on the subject under consideration.

\section{$\S 1$. The WeYL FunCTiON}

Our goal in the present section is to construct the Weyl function $m(z)$ and to prove Theorem 7 .

Let $g(z)$ be an arbitrary solution of system (7). We have

$$
\left(\begin{array}{c}
z^{-1} \alpha_{l+1} \\
z \beta_{l+1}
\end{array}\right)=\left(\begin{array}{c}
w_{l} \alpha_{l}+v_{l} \beta_{l} \\
\bar{w}_{l} \beta_{l}+\bar{v}_{l} \alpha_{l}
\end{array}\right), \quad \text { where }\left(\begin{array}{c}
\alpha_{l} \\
\beta_{l}
\end{array}\right) \stackrel{\text { def }}{=} g_{l} \text {. }
$$

Consequently,

$$
\begin{aligned}
& |z|^{-2}\left|\alpha_{l+1}\right|^{2}-|z|^{2}\left|\beta_{l+1}\right|^{2}=\left|w_{l} \alpha_{l}+v_{l} \beta_{l}\right|^{2}-\left|\bar{w}_{l} \beta_{l}+\bar{v}_{l} \alpha_{l}\right|^{2} \\
& \quad=\left(\left|w_{l}\right|^{2}-\left|v_{l}\right|^{2}\right)\left|\alpha_{l}\right|^{2}-\left(\left|w_{l}\right|^{2}-\left|v_{l}\right|^{2}\right)\left|\beta_{l}\right|^{2}=\left|\alpha_{l}\right|^{2}-\left|\beta_{l}\right|^{2} .
\end{aligned}
$$


As a result, we obtain the identity

$$
|z|^{-2}\left|\alpha_{l+1}\right|^{2}-|z|^{2}\left|\beta_{l+1}\right|^{2}=\left|\alpha_{l}\right|^{2}-\left|\beta_{l}\right|^{2} .
$$

Lemma 5. For each solution $g(z)$ of system (7), we have

$$
\left(1-|z|^{2}\right) \sum_{k=1}^{l}\left[|z|^{-2}\left|\alpha_{k}\right|^{2}+\left|\beta_{k}\right|^{2}\right]=\left|\alpha_{0}\right|^{2}-\left|\beta_{0}\right|^{2}-\left|\alpha_{l}\right|^{2}+\left|\beta_{l}\right|^{2} .
$$

For simplicity, we introduce the notation

$$
\operatorname{Sm}_{l}(g(z)) \stackrel{\text { def }}{=}\left(1-|z|^{2}\right) \sum_{k=1}^{l}\left[|z|^{-2}\left|\alpha_{k}\right|^{2}+\left|\beta_{k}\right|^{2}\right], \quad \operatorname{Sm}_{0}(g(z)) \stackrel{\text { def }}{=} 0 .
$$

Proof. Using (1.1), we obtain

$$
\begin{aligned}
\left|\alpha_{0}\right|^{2} & -\left|\beta_{0}\right|^{2}-\left|\alpha_{l}\right|^{2}+\left|\beta_{l}\right|^{2}=\left(\left|\alpha_{0}\right|^{2}-\left|\alpha_{l}\right|^{2}\right)-\left(\left|\beta_{0}\right|^{2}-\left|\beta_{l}\right|^{2}\right) \\
& =\sum_{k=1}^{l}\left(\left|\alpha_{k-1}\right|^{2}-\left|\alpha_{k}\right|^{2}\right)-\sum_{k=1}^{l}\left(\left|\beta_{k-1}\right|^{2}-\left|\beta_{k}\right|^{2}\right) \\
& =\sum_{k=1}^{l}\left(\left|\alpha_{k-1}\right|^{2}-\left|\beta_{k-1}\right|^{2}\right)-\sum_{k=1}^{l}\left(\left|\alpha_{k}\right|^{2}-\left|\beta_{k}\right|^{2}\right) \\
& =\sum_{k=1}^{l}\left(|z|^{-2}\left|\alpha_{k}\right|^{2}-|z|^{2}\left|\beta_{k}\right|^{2}\right)-\sum_{k=1}^{l}\left(\left|\alpha_{k}\right|^{2}-\left|\beta_{k}\right|^{2}\right)=\operatorname{Sm}_{l}(g(z)) .
\end{aligned}
$$

The lemma is proved.

We denote by $\|\cdot\|$ the norm of a vector in $\mathbb{C}^{2}$.

Lemma 6. For every solution $g(z)$ of system (7), the following estimate is valid for $0<|z|<1$ :

$$
\frac{|z|^{2}}{1-|z|^{2}} \operatorname{Sm}_{l}(g(z)) \leq \sum_{k=1}^{l}\left\|g_{k}(z)\right\|^{2} \leq \frac{1}{1-|z|^{2}} \operatorname{Sm}_{l}(g(z)) .
$$

Proof. This follows from the obvious inequalities

$$
\sum_{k=1}^{l}\left(\left|\alpha_{k}\right|^{2}+|z|^{2}\left|\beta_{k}\right|^{2}\right) \leq \sum_{k=1}^{l}\left(\left|\alpha_{k}\right|^{2}+\left|\beta_{k}\right|^{2}\right) \leq \sum_{k=1}^{l}\left(|z|^{-2}\left|\alpha_{k}\right|^{2}+\left|\beta_{k}\right|^{2}\right) .
$$

Consider the solutions $\theta(z)$ and $\varphi(z)$ of system (7) that satisfy the initial conditions $\theta_{0}(z)=\left(\begin{array}{l}1 \\ 0\end{array}\right)$ and $\varphi_{0}(z)=\left(\begin{array}{l}0 \\ 1\end{array}\right)$ (see the preceding section). For the coordinates of $\theta_{l}(z)$ and $\varphi_{l}(z)$, we use the notation

$$
\theta_{l}(z)=\left(\begin{array}{l}
s_{l} \\
t_{l}
\end{array}\right), \quad \varphi_{l}(z)=\left(\begin{array}{l}
e_{l} \\
h_{l}
\end{array}\right) .
$$

To construct the Weyl function $m(z)$, we consider the sequence of vectors $f_{l}(z)=\theta_{l}(z)+$ $m(z) \varphi_{l}(z)$. The next step reduces to the search for a function $m(z)$ such that the element $f(z)=\left\{f_{l}(z)\right\}_{l=0}^{\infty}$ belongs to the space $l^{2}\left(\mathbb{Z}_{+}, \mathbb{C}^{2}\right)$.

Applying Lemma 5 to $f(z)$, we obtain

$$
\operatorname{Sm}_{l}(f(z))=1-|m(z)|^{2}+\left|t_{l}+m(z) h_{l}\right|^{2}-\left|s_{l}+m(z) e_{l}\right|^{2} .
$$

Lemma 6 yields the following estimates for $0<|z|<1$ :

$$
\frac{|z|^{2}}{1-|z|^{2}} \operatorname{Sm}_{l}(f(z)) \leq \sum_{k=1}^{l}\left\|f_{k}\right\|^{2} \leq \frac{1}{1-|z|^{2}} \operatorname{Sm}_{l}(f(z)) .
$$


Thus, the relation $f(z) \in l^{2}\left(\mathbb{Z}_{+}, \mathbb{C}^{2}\right)$ is equivalent to the inequality $\operatorname{Sm}_{l}(f(z)) \leq C_{1}(z)<$ $\infty$ for $0<|z|<1$, with some $C_{1}(z)$ independent of $l$.

We construct a function $m(z)$ satisfying the condition

$$
\left|t_{l}+m(z) h_{l}\right|^{2}-\left|s_{l}+m(z) e_{l}\right|^{2} \leq 0, \quad l=0,1, \ldots .
$$

It is easily seen that condition (1.4) implies the estimate $\operatorname{Sm}_{l}(f(z)) \leq 1$. Therefore, by (1.3), $f(z) \in l^{2}\left(\mathbb{Z}_{+}, \mathbb{C}^{2}\right)$. We consider the collection of sets

$$
D_{l}(z)=\left\{\zeta:\left|t_{l}(z)+\zeta h_{l}(z)\right|^{2}-\left|s_{l}(z)+\zeta e_{l}(z)\right|^{2} \leq 0\right\}, \quad l=0,1, \ldots ;
$$

obviously, these sets are closed. We shall drop $z$ in the notation $D_{l}(z)$ if this does not lead to confusion.

Lemma 7. We have $D_{l+1} \subset D_{l} \subset\{\zeta:|\zeta|<1\}$ for $l=1,2, \ldots$.

Proof. We prove the inclusions $D_{l+1} \subset D_{l}$ by contradiction. Assume that there exists $l$ and a point $\zeta_{0}$ such that $\zeta_{0} \in D_{l+1} \backslash D_{l}$. Then

$$
\begin{aligned}
& \operatorname{Sm}_{l+1}(f(z))=1-\left|\zeta_{0}\right|^{2}+\left|t_{l+1}+\zeta_{0} h_{l+1}\right|^{2}-\left|s_{l+1}+\zeta_{0} e_{l+1}\right|^{2} \leq 1-\left|\zeta_{0}\right|^{2} \\
& \quad<1-\left|\zeta_{0}\right|^{2}+\left|t_{l}+\zeta_{0} h_{l}\right|^{2}-\left|s_{l}+\zeta_{0} e_{l}\right|^{2}=\operatorname{Sm}_{l}(f(z)) .
\end{aligned}
$$

Thus, $\operatorname{Sm}_{l+1}(f(z))<\operatorname{Sm}_{l}(f(z))$. However, by definition, the sequence $\operatorname{Sm}_{l}(f(z))$ is strictly monotone increasing. This contradiction proves the required inclusions.

The inclusions $D_{l} \subset\{\zeta:|\zeta|<1\}$ are also proved by contradiction. We assume that there exists $l \geq 1$ and a point $\zeta_{0}$ such that $\zeta_{0} \in D_{l} \backslash\{\zeta:|\zeta|<1\}$. Then

$$
0<\operatorname{Sm}_{l}(f(z))=1-\left|\zeta_{0}\right|^{2}+\left|t_{l}+\zeta_{0} h_{l}\right|^{2}-\left|s_{l}+\zeta_{0} e_{l}\right|^{2} \leq 1-\left|\zeta_{0}\right|^{2} \leq 0,
$$

a contradiction.

Lemma 8. Let $f^{1}$ and $f^{2}$ be solutions of system (7) belonging to $l^{2}\left(\mathbb{Z}_{+}, \mathbb{C}^{2}\right)$. Then $f^{1}$ and $f^{2}$ are linearly dependent.

Proof. The matrix $\left(f_{l}^{1}, f_{l}^{2}\right)$ formed by the vectors $f_{l}^{1}$ and $f_{l}^{2}$ satisfies the relation

$$
\left(f_{l+1}^{1}, f_{l+1}^{2}\right)=z^{\sigma_{3}} W_{l}\left(f_{l}^{1}, f_{l}^{2}\right) .
$$

The condition $\operatorname{det}\left(z^{\sigma_{3}} W_{l}\right)=1$ implies

$$
\operatorname{det}\left(f_{l+1}^{1}, f_{l+1}^{2}\right)=\operatorname{det}\left(z^{\sigma_{3}} W_{l}\right) \operatorname{det}\left(f_{l}^{1}, f_{l}^{2}\right)=\operatorname{det}\left(f_{l}^{1}, f_{l}^{2}\right)=C_{2} \neq C_{2}(l) .
$$

Since $f^{j} \in l^{2}\left(\mathbb{Z}_{+}, \mathbb{C}^{2}\right), j=1,2$, the limits $\lim _{l \rightarrow \infty} f_{l}^{j}$ exist and are equal to 0 . Using (1.5), we obtain $\operatorname{det}\left(f_{l}^{1}, f_{l}^{2}\right)=0$, which implies that the vectors $f^{1}$ and $f^{2}$ are linearly dependent.

Lemma 9. The set $\bigcap_{l \geq 0} D_{l}$ is nonempty and consists of a unique point.

Proof. The existence of a point $\zeta_{0} \in \bigcap_{l \geq 0} D_{l}$ follows from the fact that the sets $D_{l}$ are closed and the space of complex numbers is complete. To prove uniqueness, we argue by contradiction. Let $\zeta_{j} \in \bigcap_{l \geq 0} D_{l}, j=0,1$, and let $\zeta_{0} \neq \zeta_{1}$. Then $f^{j}=\theta+\zeta_{j} \varphi \in l^{2}\left(\mathbb{Z}_{+}, \mathbb{C}^{2}\right)$ for $j=0,1$ and, by Lemma 8 , the $f^{j}$ are linearly dependent. Putting $l=0$, we see that $\zeta_{0}=\zeta_{1}$.

We represent condition (1.4) in the form $\left|t_{l}(z)+m(z) h_{l}(z)\right| /\left|s_{l}(z)+m(z) e_{l}(z)\right| \leq 1$ and consider the mapping

$$
w=\frac{t_{l}(z)+m(z) h_{l}(z)}{s_{l}(z)+m(z) e_{l}(z)} .
$$


The relation $s_{l} h_{l}-t_{l} e_{l}=\operatorname{det}\left(\theta_{l}, \varphi_{l}\right)=1$ implies the existence of the inverse mapping, which is given by

$$
m(z, l, w)=-\frac{t_{l}(z)-s_{l}(z) w}{h_{l}(z)-e_{l}(z) w} .
$$

Consequently,

$$
D_{l}(z)=\bigcup_{|w| \leq 1}\{m(z, l, w)\} .
$$

Therefore, Lemmas 7 and 9 imply that the $\operatorname{limit}_{l i m} \lim _{l \rightarrow \infty} m(z, l, w)$ exists and is independent of $w$. We denote this limit by $m(z)$.

Lemma 10. For $0<|z|<1$, the function $m(z)$ is analytic and satisfies $|m(z)|<1$.

Proof. First, we prove that for all $l$ the function $m(z, l, w)$ is continuous in two variables $(z, w)$ in the region $\{z: 0<|z|<1\} \times\{w:|w| \leq 1\}$ and analytic in $z$ in the region $\{z: 0<|z|<1\}$. Indeed, relation (1.1) implies the inequality $\left|h_{l}(z)\right|>\left|e_{l}(z)\right|$, which, in turn, implies the estimate

$$
\left|h_{l}(z)-e_{l}(z) w\right|>0, \quad(z, w) \in\{z: 0<|z|<1\} \times\{w:|w| \leq 1\},
$$

valid for all $l$. The definition of $\theta_{l}(z)$ and $\varphi_{l}(z)$ shows that the functions $t_{l}(z), s_{l}(z)$, $h_{l}(z)$, and $e_{l}(z)$ are analytic in the region $0<|z|<1$. Taking (1.6) and (1.7) into account, we obtain the required statement.

Now, we prove that the function $m(z)$ is continuous in the region $0<|z|<1$. For this, we fix a point $z_{0}$ and an arbitrary $\varepsilon>0$. By Lemmas 7 and 9 , there exists $l_{0}$ such that diam $D_{l_{0}}\left(z_{0}\right)<\varepsilon / 2$. The continuity of $m\left(z, l_{0}, w\right)$ in the variables $(z, w)$ implies the existence of a number $\delta>0$ such that

$$
\operatorname{diam} \bigcup_{\left|z-z_{0}\right|<\delta} D_{l_{0}}(z)<\varepsilon
$$

This fact can be proved by contradiction. Indeed, assume that there exist sequences $z_{j}^{1}$, $w_{j}^{1}$ and $z_{j}^{2}, w_{j}^{2}$ such that $\lim _{j \rightarrow \infty} z_{j}^{1}=\lim _{j \rightarrow \infty} z_{j}^{2}=z_{0}$ and

$$
\left|m\left(z_{j}^{1}, l_{0}, w_{j}^{1}\right)-m\left(z_{j}^{2}, l_{0}, w_{j}^{2}\right)\right| \geq \varepsilon .
$$

Since the $w_{j}^{1}$ belong to the compact set $\{w:|w| \leq 1\}$, there exists a subsequence $w_{j_{l}}^{1}$ converging to some $w^{1}$. Similarly, there exists a subsequence $w_{j_{l_{k}}}^{2}$ of $w_{j_{l}}^{2}$ converging to some $w^{2}$. For simplicity, we preserve the notation $w_{i}^{1}$ and $w_{i}^{2}$ for the subsequences $w_{j_{l_{i}}}^{1}$ and $w_{j_{l}}^{2}$. Since $m\left(z, l_{0}, w\right)$ is continuous, there exists $\delta>0$ such that $\mid m\left(z_{j}^{k}, l_{0}, w_{j}^{k}\right)-$ $m\left(z_{0}, l_{0}, w^{k}\right) \mid<\varepsilon / 4$ for $\left|z_{j}^{k}-z^{0}\right|+\left|w_{j}^{k}-w^{k}\right|<\delta$, where $k=1,2$. Moreover, since $\operatorname{diam} D_{l_{0}}\left(z_{0}\right)<\varepsilon / 2$, we have $\left|m\left(z_{0}, l_{0}, w^{1}\right)-m\left(z_{0}, l_{0}, w^{2}\right)\right|<\varepsilon / 2$. If $\left|z_{j}^{k}-z^{0}\right|+\left|w_{j}^{k}-w^{k}\right|<$ $\delta$, then

$$
\begin{aligned}
& \left|m\left(z_{j}^{1}, l_{0}, w_{j}^{1}\right)-m\left(z_{j}^{2}, l_{0}, w_{j}^{2}\right)\right| \\
& \quad \leq\left|m\left(z_{j}^{1}, l_{0}, w_{j}^{1}\right)-m\left(z_{0}, l_{0}, w^{1}\right)\right| \\
& \quad+\left|m\left(z_{0}, l_{0}, w^{1}\right)-m\left(z_{0}, l_{0}, w^{2}\right)\right|+\left|m\left(z_{0}, l_{0}, w^{2}\right)-m\left(z_{j}^{2}, l_{0}, w_{j}^{2}\right)\right| \\
& \quad<\varepsilon
\end{aligned}
$$

which contradicts (1.9).

Lemmas 7 and 9 show that $m(z) \in D_{l_{0}}(z)$. Therefore,

$$
m(z) \in \bigcup_{\left|z-z_{0}\right|<\delta} D_{l_{0}}(z), \quad z \in\left\{z:\left|z-z_{0}\right|<\delta\right\} .
$$


Now, recalling (1.8), we see that $\left|m(z)-m\left(z_{0}\right)\right|<\varepsilon$ for $\left|z-z_{0}\right|<\delta$. Thus, $m(z)$ is a continuous function for $0<|z|<1$.

We fix an arbitrary $w$ (say, $w=0$ ) and prove that the limit $m(z)=\lim _{l \rightarrow \infty} m(z, l, 0)$ is attained uniformly on every compact subset $K \subset\{z: 0<|z|<1\}$. Assume the contrary. Then there exist $\varepsilon>0$, a sequence $l_{j} \rightarrow \infty$, and points $z_{j}$ in $K$ such that

$$
\left|m\left(z_{j}\right)-m\left(z_{j}, l_{j}, 0\right)\right|>\varepsilon \text {. }
$$

Since $K$ is compact, there is a subsequence $z_{j_{k}}$ that converges to some $z^{1} \in K$. Since $m(z)$ is continuous, for some $\delta_{1}>0$ we have

$$
\left|m(z)-m\left(z^{1}\right)\right|<\varepsilon / 2 \quad \text { for }\left|z-z^{1}\right|<\delta_{1} .
$$

As above, there exists $l^{1}$ such that $\operatorname{diam} D_{l^{1}}\left(z^{1}\right)<\varepsilon / 4$, and $\operatorname{diam} \bigcup_{\left|z-z^{1}\right|<\delta_{2}} D_{l^{1}}(z)<\varepsilon / 2$ for some $\delta_{2}>0$. Since the sequence $z_{j_{k}}$ converges to $z^{1}$, there exists $C_{3}$ such that $\left|z_{j_{k}}-z^{1}\right|<\min \left(\delta_{1}, \delta_{2}\right)$ for $k \geq C_{3}$. Now, let $k \geq C_{3}$; then

$$
m\left(z_{j_{k}}, l_{j_{k}}, 0\right) \in D_{l_{j_{k}}}\left(z_{j_{k}}\right) \subset \bigcup_{\left|z-z^{1}\right|<\delta_{2}} D_{l_{j_{k}}}(z) \subset \bigcup_{\left|z-z^{1}\right|<\delta_{2}} D_{l^{1}}(z) .
$$

Consequently,

$$
\left|m\left(z^{1}\right)-m\left(z_{j_{k}}, l_{j_{k}}, 0\right)\right|<\varepsilon / 2 \quad \text { for } k \geq C_{3} .
$$

Combining (1.11) and (1.12), we obtain

$$
\left|m\left(z_{j_{k}}\right)-m\left(z_{j_{k}}, l_{j_{k}}, 0\right)\right| \leq\left|m\left(z_{j_{k}}\right)-m\left(z^{1}\right)\right|+\left|m\left(z^{1}\right)-m\left(z_{j_{k}}, l_{j_{k}}, 0\right)\right| \leq \varepsilon,
$$

which contradicts (1.10), thus proving the required uniformity.

Since $m(z, l, 0)$ is analytic and the limit $m(z)=\lim _{l \rightarrow \infty} m(z, l, 0)$ is uniform, we conclude that $m(z)$ is analytic for $0<|z|<1$. The estimate $|m(z)|<1$ follows from Lemma 7.

Proof of Theorem 7. Since the function $m(z)$ is analytic and bounded in the annulus $0<|z|<1$ (see Lemma 10), it has a removable singularity at $z=0$. Consequently, $m(z)$ admits analytic continuation to the region $|z|<1$. The maximum principle implies that $|m(z)|<1$ everywhere in the disk $|z|<1$. Since $m(z)$ satisfies (1.4) for all $l$, Lemmas 5 and 6 imply that $f(z) \in l^{2}\left(\mathbb{Z}_{+}, \mathbb{C}^{2}\right)$ for $0<|z|<1$. The theorem is proved.

\section{§2. A BFZ-TYPE SPECTRAL IDENTITY}

In the present section, we assume that $W_{l} \equiv I$ for $l \geq l_{0}$, with some $l_{0}$. For such $W_{l}$, there is a solution $h$ of system (7) satisfying the condition $h_{l}=\left(\begin{array}{c}z^{l} \\ 0\end{array}\right)$ for $l \geq l_{0}$. We use the notation

$$
\left(\begin{array}{l}
a(z) \\
b(z)
\end{array}\right)=h_{0}(z), \quad\left(\begin{array}{c}
\alpha_{l}(z) \\
\beta_{l}(z)
\end{array}\right)=h_{l}(z)
$$

Lemma 11. The function $a(z)$ is analytic and does not vanish in the region $0<|z| \leq 1$.

Proof. The analyticity of $a(z)$ follows from that of the matrix $\left(z^{\sigma_{3}} W_{l}\right)^{-1}$.

Assume that there is $z_{0}$ such that $a\left(z_{0}\right)=0$ and $0<\left|z_{0}\right| \leq 1$. Then, by (1.1), we have $\left|\alpha_{l}\left(z_{0}\right)\right|<\left|\beta_{l}\left(z_{0}\right)\right|$ for all $l$. Putting $l=l_{0}$ in this inequality, we arrive at a contradiction: $\left|z_{0}\right|^{l_{0}}<0$.

Lemma 12. The function $a(z)$ admits analytic continuation to the disk $\{z:|z| \leq 1\}$, $a(0)=\prod_{l \geq 0} \bar{w}_{l}$. 
Proof. We prove the following estimates by induction:

$$
\alpha_{l}(z)=z^{l}\left(\prod_{k \geq l} \bar{w}_{k}+O\left(z^{2}\right)\right), \quad \beta_{l}(z)=O\left(z^{l}\right), \quad l \geq 0 .
$$

For $l=l_{0}$, the claim follows from the relations $\alpha_{l}(z)=z^{l}$ and $\beta_{l}=0, l \geq l_{0}$. Now, suppose (2.1) is fulfilled for $l \geq p$. We prove that (2.1) is valid for $l=p-1$. Indeed,

$$
\begin{aligned}
\left(\begin{array}{c}
\alpha_{p-1}(z) \\
\beta_{p-1}(z)
\end{array}\right) & =W_{p-1}^{-1} z^{-\sigma_{3}}\left(\begin{array}{c}
\alpha_{p}(z) \\
\beta_{p}(z)
\end{array}\right)=\left(\begin{array}{cc}
z^{-1} \bar{w}_{p-1} & -z v_{p-1} \\
-z^{-1} \bar{v}_{p-1} & z w_{p-1}
\end{array}\right)\left(\begin{array}{c}
\alpha_{p}(z) \\
\beta_{p}(z)
\end{array}\right) \\
& =\left(\begin{array}{c}
z^{p-1} \bar{w}_{p-1}\left(\prod_{k \geq p} \bar{w}_{k}+O\left(z^{2}\right)\right)-z v_{p-1} O\left(z^{p}\right) \\
-z^{-1} \bar{v}_{p-1} O\left(z^{p}\right)+z w_{p-1} O\left(z^{p}\right)
\end{array}\right) \\
& =\left(\begin{array}{c}
z^{p-1}\left(\prod_{k \geq p} \bar{w}_{k}+O\left(z^{2}\right)\right) \\
O\left(z^{p-1}\right)
\end{array}\right) .
\end{aligned}
$$

Thus, estimates (2.1) are proved.

Putting $l=0$ in the first relation in (2.1), we obtain

$$
a(z)=\prod_{l \geq 0} \bar{w}_{l}+O\left(z^{2}\right)
$$

which implies the lemma.

Lemma 13. The function a $(z)$ satisfies the identity

$$
\int_{0}^{2 \pi} \ln \left|a\left(r e^{i \phi}\right)\right|^{2} d \phi=4 \pi \sum_{l \geq 0} \ln \left|w_{l}\right|
$$

for all $r \leq 1$.

Proof. By Lemmas 11 and 12, the function $\ln a(z)$ is analytic in the disk $\{z:|z| \leq 1\}$. The function $\ln |a(z)|$ is harmonic in the same region, being the real part of an analytic function. Applying the mean value theorem, we obtain

$$
\int_{0}^{2 \pi} \ln \left|a\left(r e^{i \phi}\right)\right|^{2} d \phi=2 \pi \ln |a(0)|^{2}=4 \pi \sum_{l \geq 0} \ln \left|w_{l}\right| .
$$

Lemma 14. Suppose that there exists $l_{0}$ such that $W_{l} \equiv I$ for $l \geq l_{0}$. Then the Weyl function of system (7) satisfies the inequality

$$
-\int_{0}^{2 \pi} \ln \left(1-\left|m\left(r e^{i \phi}\right)\right|^{2}\right) d \phi \leq 4 \pi \sum_{l \geq 0} \ln \left|w_{l}\right|
$$

for all $r \leq 1$.

Proof. By Lemma 8, the solutions $f(z)$ and $h(z)$ are linearly dependent. Putting $l=0$, we see that the vectors $\left(\begin{array}{c}1 \\ m(z)\end{array}\right)$ and $\left(\begin{array}{c}a(z) \\ b(z)\end{array}\right)$ are also linearly dependent. This implies that $a(z), b(z)$, and $m(z)$ are related by the formula

$$
m(z)=b(z) / a(z) .
$$

Applying (1.1) to $h_{l}(z)$, we obtain

$$
\begin{aligned}
& |a(z)|^{2}-|b(z)|^{2}=|z|^{-2}\left(\left|\alpha_{1}\right|^{2}-|z|^{4}\left|\beta_{1}\right|^{2}\right) \\
& \quad \geq|z|^{-2}\left(\left|\alpha_{1}\right|^{2}-\left|\beta_{1}\right|^{2}\right) \geq|z|^{-2 l_{0}}\left(\left|\alpha_{l_{0}}\right|^{2}-\left|\beta_{l_{0}}\right|^{2}\right)=1
\end{aligned}
$$

for $0<|z| \leq 1$. Thus,

$$
|a(z)|^{2}-|b(z)|^{2} \geq 1, \quad 0<|z| \leq 1 .
$$


By continuity, inequality (2.2) extends to the entire disk $|z| \leq 1$. Hence,

$$
\left(1-|m(z)|^{2}\right)^{-1} \leq|a(z)|^{2} .
$$

To conclude the proof, it remains to use Lemma 13.

In $l^{2}\left(\mathbb{Z}_{+}, \mathbb{C}^{2}\right)$ we consider the operator

$$
M g_{l}=g_{l+1}-z^{\sigma_{3}} W_{l} g_{l}, \quad 0<|z|<1, \quad l=0,1, \ldots,
$$

with domain

$$
\operatorname{dom}(M)=\left\{g: g_{0}=\left(\begin{array}{l}
0 \\
.
\end{array}\right), g \in l^{2}\left(\mathbb{Z}_{+}, \mathbb{C}^{2}\right)\right\} .
$$

We recall that $\theta(z), \varphi(z)$, and $f(z)$ are the vector-valued functions defined in Theorem 7. The kernel of the operator $M$ is trivial. Indeed, let $g$ be a nonzero solution of the equation $M g=0$. Since $g \in \operatorname{dom}(M)$, we have $g=C_{4} \varphi$ on one hand, and $g=C_{5} f$ on the other. As a result, we see that the vectors $\varphi$ and $f$ are proportional, which contradicts the definition of $f$. Since the kernel of $M$ is trivial, the operator $M$ is invertible. The inverse is given by the formula

$$
\left\{M^{-1} q\right\}_{l} \equiv M^{-1} q_{l}=\sum_{k \geq 0} G(l, k) q_{k}, \quad l=0,1, \ldots,
$$

where

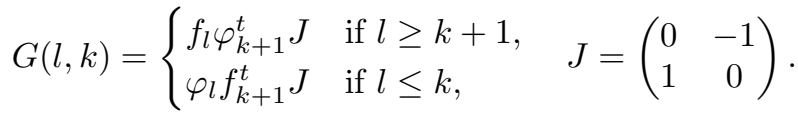

Here ${ }^{t}$ stands for transposition. The domain of $\operatorname{dom}\left(M^{-1}\right)$ is $l^{2}\left(\mathbb{Z}_{+}, \mathbb{C}^{2}\right)$.

Consider the set of matrices $W_{l}$ satisfying (9) and assume that the series (10) converges. Let $m(z)$ be the Weyl function for (7), and let $M$ be the operator constructed starting with $W_{l}$. Now, for $k \geq 1$, we consider the system (7) determined by the following set of matrices:

$$
\begin{cases}W_{l}^{k}=W_{l} & \text { if } l \leq k-1, \\ W_{l}^{k}=I & \text { if } l \geq k .\end{cases}
$$

Let $m_{k}(z)$ and $M^{k}$ denote the Weyl function and the operator of the resulting system, respectively.

Lemma 15. Suppose the limit (11) exists. Then, as $k \rightarrow \infty$, the Weyl function $m_{k}(z)$ tends to $m(z)$ uniformly on every compact subset of $\{z: 0<|z|<1\}$.

Proof. We have the resolvent identity

$$
M^{-1}-M_{k}^{-1}=M^{-1}\left(M_{k}-M\right) M_{k}^{-1} .
$$

For $k \geq 1$, we have the well-defined vector-valued function

$$
\theta_{1}(z)=z^{\sigma_{3}} W_{0}^{k} \theta_{0}=z^{\sigma_{3}} W_{0}\left(\begin{array}{l}
1 \\
0
\end{array}\right) .
$$

Consider the following element $q$ of the space $\operatorname{dom}\left(M^{-1}\right)=\operatorname{dom}\left(M_{k}^{-1}\right)$ :

$$
q=\left\{q_{l}\right\}_{l=0}^{\infty}, \quad q_{l}= \begin{cases}\theta_{1}(z) & \text { if } l=0 \\ 0 & \text { if } l \geq 1\end{cases}
$$


The following relations are valid:

$$
\begin{gathered}
\left\{M_{k}^{-1} q\right\}_{l}=G(l, 0), \quad \theta_{1}(z)=\left\{\begin{array}{ll}
m_{k}(z)\left(\begin{array}{l}
0 \\
1
\end{array}\right) & \text { if } l=0, \\
f_{l}^{k}(z) & \text { if } l \geq 1,
\end{array} \quad\left\{M^{-1} q\right\}_{0}=m(z)\left(\begin{array}{l}
0 \\
1
\end{array}\right),\right. \\
\left\{M^{-1}\left(M_{k}-M\right) M_{k}^{-1} q\right\}_{0}=\left(\begin{array}{l}
0 \\
1
\end{array}\right) \sum_{l \geq k}\left(f_{l+1}(z)\right)^{t} J z^{\sigma_{3}}\left(I-W_{l}\right) f_{l}^{k}(z) .
\end{gathered}
$$

Now, we calculate the values of both sides of $(2.6)$ at $q$. We have

$$
m(z)-m_{k}(z)=\sum_{l \geq k}\left(f_{l+1}(z)\right)^{t} J z^{\sigma_{3}}\left(I-W_{l}\right) f_{l}^{k}(z),
$$

whence

$$
\begin{aligned}
\mid m(z) & -m_{k}(z) \mid \leq \sum_{l \geq k}\left\|z^{\sigma_{3}}\right\|\left\|W_{l}-I\right\|\left\|f_{l+1}(z)\right\|\left\|f_{l}^{k}(z)\right\| \\
& \leq\left\|z^{\sigma_{3}}\right\|\left(\sum_{l \geq 1}\left\|f_{l}(z)\right\|^{2}\right)^{1 / 2}\left(\sum_{l \geq 1}\left\|f_{l}^{k}(z)\right\|^{2}\right)^{1 / 2} \sup _{l \geq k}\left\|W_{l}-I\right\| .
\end{aligned}
$$

We fix an arbitrary compact set $K \subset\{z: 0<|z|<1\}$ and put $r=\min _{z \in K}|z|>0$ and $R=\max _{z \in K}|z|<1$. Lemma 3 and relations (1.2)-(1.4) imply the inequalities

$$
\sum_{l \geq 1}\left\|f_{l}(z)\right\|^{2} \leq \frac{1}{1-R^{2}}, \quad \sum_{l \geq 1}\left\|f_{l}^{k}(z)\right\|^{2} \leq \frac{1}{1-R^{2}} .
$$

Obviously, $\left\|z^{\sigma_{3}}\right\|=1 / r$. Substituting this in (2.7), we obtain

$$
\left|m(z)-m_{k}(z)\right| \leq \frac{1}{r\left(1-R^{2}\right)} \sup _{l \geq k}\left\|W_{l}-I\right\|, \quad z \in K,
$$

which implies the lemma.

Proof of Lemma 2. By Lemma 15 and Theorem 7, we can pass to the limit under the integral sign,

$$
-\lim _{k \rightarrow \infty} \int_{0}^{2 \pi} \ln \left(1-\left|m_{k}\left(r e^{i \phi}\right)\right|^{2}\right) d \phi=-\int_{0}^{2 \pi} \ln \left(1-\left|m\left(r e^{i \phi}\right)\right|^{2}\right) d \phi
$$

for $0<r<1$. Applying Lemma 14 to the left-hand side of (2.8), we obtain

$$
-\int_{0}^{2 \pi} \ln \left(1-\left|m\left(r e^{i \phi}\right)\right|^{2}\right) d \phi \leq 4 \pi \sum_{l \geq 0} \ln \left|w_{l}\right| .
$$

It remains to observe that, by Corollary 1, we can apply the Fatou lemma to inequality (2.9) when passing to the limit as $r \rightarrow 1-0$. This yields the required estimate.

\section{§3. Some estimates}

In the present section, we prove Lemmas 3 and 4, which are needed in the proof of Theorem 2. These results are not really new; their analogs can be found in [LS].

Proof of Lemma 3. We consider the operator $M$ given by formula (2.3), together with the operator $M_{0}$ given by (2.3) with $W_{l} \equiv I$. These operators are boundedly invertible (see (2.4)). We use the resolvent identity

$$
M^{-1}-M_{0}^{-1}=M_{0}^{-1}\left(M_{0}-M\right) M^{-1} .
$$

Consequently,

$$
\left(I+M_{0}^{-1} z^{\sigma_{3}}(I-\widehat{W})\right) M^{-1}=M_{0}^{-1},
$$

where $\widehat{W}$ is the operator of multiplication by $W_{l}$. 
Let $G_{0}(l, k)$ be the Green function for $M_{0}$ (see (2.5)), and let $\varphi^{0}$ and $f^{0}$ be the corresponding solutions of system (7) with $W_{l} \equiv I$ (see $\S 1$ ). For $0<|z|<1$, simple calculations show that

$$
\begin{aligned}
& G_{0}(l, k)=\left(\begin{array}{ll}
1 & 0 \\
0 & 0
\end{array}\right) z^{l-k-1} \quad \text { if } l \geq k+1, \\
& G_{0}(l, k)=\left(\begin{array}{cc}
0 & 0 \\
0 & -1
\end{array}\right) z^{k-l+1} \quad \text { if } l \leq k .
\end{aligned}
$$

In particular,

$$
\left\|G_{0}(l, k)\right\| \leq|z|^{|l-k-1|}, \quad\left\|G_{0}(l, k) z^{\sigma_{3}}\right\| \leq|z|^{|l-k|} .
$$

Invoking Schur's criterion, we obtain

$$
\left\|M_{0}^{-1} z^{\sigma_{3}}\right\| \leq \sup _{l \geq 0} \sum_{k=0}^{\infty}|z|^{|l-k|} \leq \sum_{k \in \mathbb{Z}}|z|^{|k|} \leq \frac{2}{1-|z|}, \quad\left\|M_{0}^{-1}\right\| \leq \frac{2}{1-|z|} .
$$

Estimates (13) and (3.2) imply that

$$
\begin{gathered}
\left\|M_{0}^{-1} z^{\sigma_{3}}(I-\widehat{W})\right\| \leq\left\|M_{0}^{-1} z^{\sigma_{3}}\right\|\|(I-\widehat{W})\| \\
\leq \frac{1}{4(1-|z|)} \leq \frac{1}{2} \quad \text { for } 0<|z| \leq \frac{1}{2} .
\end{gathered}
$$

Consequently, for $0<|z| \leq 1 / 2$ the operator $I+M_{0}^{-1} z^{\sigma_{3}}(I-\widehat{W})$ is boundedly invertible. By (3.1) and (3.2),

$$
\begin{aligned}
\left\|M^{-1}\right\| & \leq\left\|\left(I+M_{0}^{-1} z^{\sigma_{3}}(I-\widehat{W})\right)^{-1}\right\|\left\|M_{0}^{-1}\right\| \leq 2\left\|M_{0}^{-1}\right\| \\
& \leq \frac{4}{1-|z|} \leq 8 \quad \text { for } 0<|z| \leq \frac{1}{2}
\end{aligned}
$$

The norm of the matrix $G(l, l-1, z)$ can be estimated in terms of the norm of the operator $M^{-1}$. Indeed,

$$
\begin{aligned}
\|G(l, l-1)\|^{2} \leq \sum_{k=0}^{\infty}\|G(k, l-1)\|^{2} & =\sum_{k=0}^{\infty}\left\|\sum_{n=0}^{\infty} G(k, n) \delta_{l-1}^{n}\right\|^{2} \\
\leq \sup _{\|q\|=1} \sum_{k=0}^{\infty}\left\|\sum_{n=0}^{\infty} G(k, n) q\right\|^{2} & =\sup _{\|q\|=1}\left\|M^{-1} q\right\|^{2}=\left\|M^{-1}\right\|^{2} .
\end{aligned}
$$

Consequently,

$$
\|G(l, l-1, z)\| \leq\left\|M^{-1}\right\| \leq 8 \quad \text { for } 0<|z| \leq \frac{1}{2} .
$$

The functions $f(z)$ and $\varphi(z)$ are analytic in the annulus $0<|z|<1$. Therefore, representation (2.5) for the matrix $G(l, l-1, z)$ implies that this matrix is analytic in the same region. By (3.3), the matrix $G(l, l-1, z)$ admits analytic continuation to the disk $|z|<1$. By the mean-value theorem,

$$
\int_{0}^{2 \pi} G\left(l, l-1, r e^{i \phi}\right) d \phi=2 \pi G(l, l-1,0), \quad r<1 .
$$

By Corollary 1 and Fatou's lemma, in (3.4) we can pass to the limit as $r \rightarrow 1$, which yields

$$
\left\|\int_{0}^{2 \pi} G\left(l, l-1, r e^{i \phi}\right) d \phi\right\| \leq 16 \pi
$$


From property (8) it follows that $\theta=\sigma_{1} \bar{\varphi}$ for $z=e^{i \phi}$. We denote by $\varphi_{l}^{1}$ and $\varphi_{l}^{2}$ the components of the vector $\varphi_{l}$. Applying (2.5), we obtain

$$
\begin{aligned}
G(l, l & \left.-1, e^{i \phi}\right)=\left(\sigma_{1} \bar{\varphi}_{l}+m\left(e^{i \phi}\right) \varphi_{l}\right) \varphi_{l}^{t} J \\
& =\left(\left(\begin{array}{cc}
\varphi_{l}^{1} \bar{\varphi}_{l}^{2} & \left|\varphi_{l}^{2}\right|^{2} \\
\left|\varphi_{l}^{1}\right|^{2} & \varphi_{l}^{2} \bar{\varphi}_{l}^{1}
\end{array}\right)+m\left(e^{i \phi}\right)\left(\begin{array}{cc}
\left(\varphi_{l}^{1}\right)^{2} & \varphi_{l}^{1} \varphi_{l}^{2} \\
\varphi_{l}^{1} \varphi_{l}^{2} & \left(\varphi_{l}^{2}\right)^{2}
\end{array}\right)\right) J \\
& =\left(\begin{array}{cc}
\left|\varphi_{l}^{2}\right|^{2}+m\left(e^{i \phi}\right) \varphi_{l}^{1} \varphi_{l}^{2} & -\varphi_{l}^{1} \bar{\varphi}_{l}^{2}-m\left(e^{i \phi}\right)\left(\varphi_{l}^{1}\right)^{2} \\
\varphi_{l}^{2} \bar{\varphi}_{l}^{1}+m\left(e^{i \phi}\right)\left(\varphi_{l}^{2}\right)^{2} & -\left|\varphi_{l}^{1}\right|^{2}-m\left(e^{i \phi}\right) \varphi_{l}^{1} \varphi_{l}^{2}
\end{array}\right) .
\end{aligned}
$$

We subtract the diagonal entries in (3.6) and use estimate (3.5). As a result, we see that

$$
\int_{0}^{2 \pi}\left(1-\left|m\left(e^{i \phi}\right)\right|\right)\left\|\varphi_{l}\left(e^{i \phi}\right)\right\|^{2} d \phi \leq 32 \pi
$$

For $z=e^{i \phi}$ we have $F_{l}=\left(\sigma_{1} \bar{\varphi}_{l}, \varphi_{l}\right)$. Now, estimate (3.7) can be transformed to

$$
\int_{0}^{2 \pi}\left(1-\left|m\left(e^{i \phi}\right)\right|\right)\left\|F_{l}\left(e^{i \phi}\right)\right\|^{2} d \phi \leq 64 \pi
$$

which allows us to write

$$
\begin{aligned}
& \int_{0}^{2 \pi}\left(1-\left|m\left(e^{i \phi}\right)\right|\right) \sum_{l=0}^{L} a_{l}\left\|F_{l}\right\|\left\|F_{l+1}\right\| d \phi \\
& \quad \leq \frac{1}{2} \int_{0}^{2 \pi}\left(1-\left|m\left(e^{i \phi}\right)\right|\right) \sum_{l=0}^{L} a_{l}\left\|F_{l}\right\|^{2} d \phi+\frac{1}{2} \int_{0}^{2 \pi}\left(1-\left|m\left(e^{i \phi}\right)\right|\right) \sum_{l=0}^{L} a_{l}\left\|F_{l+1}\right\|^{2} d \phi \\
& \quad \leq 64 \pi \sum_{l=0}^{\infty} a_{l} .
\end{aligned}
$$

Applying Beppo Levi's theorem and recalling that $\left|m\left(e^{i \phi}\right)\right|<1$ for almost all $\phi \in[0,2 \pi)$ (see Lemma 2), we conclude that the series $\sum_{l=0}^{\infty} a_{l}\left\|F_{l}\right\|\left\|F_{l+1}\right\|$ converges almost everywhere with respect to Lebesgue measure.

The second estimate in Lemma 3 follows from the inequalities

$$
\begin{aligned}
\int_{0}^{2 \pi} & \left(1-\left|m\left(e^{i \phi}\right)\right|\right) \liminf _{L \rightarrow \infty} \frac{1}{L} \sum_{l=0}^{L}\left\|F_{l}\right\|^{2} d \phi \\
& \leq \liminf _{L \rightarrow \infty} \frac{1}{L} \sum_{l=0}^{L} \int_{0}^{2 \pi}\left(1-\left|m\left(e^{i \phi}\right)\right|\right)\left\|F_{l}\right\|^{2} d \phi \leq 64 \pi
\end{aligned}
$$

and the estimate $\left|m\left(e^{i \phi}\right)\right|<1$, which is valid for almost all $\phi \in[0,2 \pi)$ (see Lemma 2).

Proof of Lemma 4. Let $u$ and $v$ be two linearly independent solutions of system (14). To simplify calculation, we assume that $\operatorname{det}\left(u_{0}, v_{0}\right)=1$. This can always be ensured by multiplying one of the solutions by an appropriate constant. Since $\operatorname{det} K_{l}=1$, we have

$$
\operatorname{det}\left(u_{l}, v_{l}\right)=\operatorname{det}\left(u_{0}, v_{0}\right)=1,
$$

whence

$$
1 \leq\left\|u_{l}\right\|\left\|v_{l}\right\| .
$$

Summing from 0 to $L$, we obtain

$$
1 \leq\left(\frac{1}{L} \sum_{l=0}^{L}\left\|u_{l}\right\|\left\|v_{l}\right\|\right)^{2} \leq\left(\frac{1}{L} \sum_{l=0}^{L}\left\|u_{l}\right\|^{2}\right)\left(\frac{1}{L} \sum_{l=0}^{L}\left\|v_{l}\right\|^{2}\right) .
$$


Since $u_{l}=B_{l} u_{0}$, we have

$$
\frac{\sum_{l=0}^{L}\left\|u_{l}\right\|^{2}}{\sum_{l=0}^{L}\left\|v_{l}\right\|^{2}}=\frac{1}{L} \frac{\sum_{l=0}^{L}\left\|u_{l}\right\|^{2}}{\frac{1}{L} \sum_{l=0}^{L}\left\|v_{l}\right\|^{2}} \leq\left(\frac{1}{L} \sum_{l=0}^{L}\left\|u_{l}\right\|^{2}\right)^{2} \leq\left\|u_{0}\right\|^{4}\left(\frac{1}{L} \sum_{l=0}^{L}\left\|B_{l}\right\|^{2}\right)^{2} .
$$

Now, let $v_{l}$ be a subordinate solution. By the assumptions of the lemma,

$$
\liminf _{L \rightarrow \infty} \frac{1}{L} \sum_{l=0}^{L}\left\|B_{l}\right\|^{2}<\infty
$$

Therefore, by (3.9), there exists a subsequence $L_{j}$ such that

$$
\frac{\sum_{l=0}^{L_{j}}\left\|u_{l}\right\|^{2}}{\sum_{l=0}^{L_{j}}\left\|v_{l}\right\|^{2}} \leq C_{10}<\infty, \quad j=1,2, \ldots,
$$

which contradicts the definition of a subordinate solution.

\section{$\S 4$. Proof of Theorem 2}

The main difficulty arising in the proof of Theorem 2 is that the matrices $S_{l}$ occurring in system (5) may depend on the spectral parameter $E$, and Theorem 6 cannot be applied to system (5) directly. Nevertheless, we know that, for large $l$, the matrices $S_{l}$ are independent of $E$. Therefore, from (5) we can extract a new, unperturbed system (15) satisfying the assumptions of Theorem 6 . Moreover, the unperturbed system (15) differs from (5) by a small correction, which may depend on $E$. Since this correction is small, we can prove that the solutions of the two systems are close to each other, and this will allow us to apply the results of Theorem 6 to system (5).

First, we choose $W_{l}$ in (7) so as to be able to apply Theorem 6 and Lemma 3 to system (7). Since the Fourier coefficients $r(l)$ are bounded, we have $\lim _{l \rightarrow \infty} r(l) l^{-1 / 2}=0$. Therefore, there exists a constant $L_{1}$ such that

$$
|r(l)| l^{-1 / 2} \leq 1 / 16, \quad l \geq L_{1} .
$$

Now, we consider system (5) with matrices

$$
\begin{aligned}
& W_{l}=I \quad \text { for } 0 \leq l<L_{1}, \\
& W_{l}=\left(\begin{array}{cc}
\sqrt{1+|r(l)|^{2} l^{-1}} & r(l) l^{-1 / 2} e^{\frac{2 i}{3 F}(\pi l)^{3}} \\
\bar{r}(l) l^{-1 / 2} e^{-\frac{2 i}{3 F}(\pi l)^{3}} & \sqrt{1+|r(l)|^{2} l^{-1}}
\end{array}\right), \quad \text { where } l \geq L_{1} .
\end{aligned}
$$

It is easily seen that

$$
\left\|W_{l}-I\right\| \leq 1 / 8 \text { for } l=0,1, \ldots .
$$

We rewrite systems (5) and (15) in the matrix form:

$$
\begin{gathered}
B_{l+1}=e^{i \phi \sigma_{3}}\left(W_{l}+V_{l}\right) B_{l}, \quad B_{0}=I, \quad \phi=\pi \frac{E}{F}, \\
F_{l+1}=e^{i \phi \sigma_{3}} W_{l} F_{l}, \quad F_{0}=I .
\end{gathered}
$$

Here the matrices $S_{l}$ are represented as $S_{l}=W_{l}+V_{l}$, where the $W_{l}$ are defined in (4.1).

Theorem 9. If Assumption (C) is fulfilled, then system (4.2) has no subordinate solutions for almost any $\phi \in[0,2 \pi)$ with respect to Lebesgue measure.

Proof. By Assumption (C), the series

$$
\sum_{l=0}^{\infty}\left\|V_{l}\right\|=\sum_{l=0}^{\infty}\left\|S_{l}-W_{l}\right\|
$$


converges. The matrices $W_{l}$ satisfy $\operatorname{det} W_{l}=1$. Therefore, $\operatorname{det} F_{l}=1$ for all $l \geq 0$. It follows that the $F_{l}$ are invertible, and $\left\|F_{l}\right\|=\left\|F_{l}^{-1}\right\|$ for all $l \geq 0$. Using the substitution

$$
B_{l}=F_{l} U_{l}, \quad U_{0}=I \text {, }
$$

we reshape (4.2) to

$$
U_{l+1}=\left(I+F_{l+1}^{-1} e^{i \phi \sigma_{3}} V_{l} F_{l}\right) U_{l} .
$$

To system (4.3), we can apply Lemma 3 with $a_{l}=\left\|V_{l}\right\|$. By that lemma, for almost all $\phi \in[0,2 \pi)$ we have

$$
\left\|U_{l}\right\| \leq \prod_{k=0}^{l-1}\left(I+\left\|F_{l+1}^{-1}\right\|\left\|V_{l}\right\|\left\|F_{l}\right\|\right) \leq \exp \frac{3}{2}\left(I+\left\|V_{l}\right\|\left\|F_{l+1}\right\|\left\|F_{l}\right\|\right) \leq e^{\frac{3}{2} C_{9}(\phi)} .
$$

Again by Lemma 3,

$$
\begin{aligned}
\liminf _{L \rightarrow \infty} & \frac{1}{L} \sum_{l=0}^{L}\left\|B_{l}\right\|^{2} \leq \liminf _{L \rightarrow \infty} \frac{1}{L} \sum_{l=0}^{L}\left\|F_{l}\right\|^{2}\left\|U_{l}\right\|^{2} \\
& \leq e^{3 C_{9}(\phi)} \liminf _{L \rightarrow \infty} \frac{1}{L} \sum_{l=0}^{L}\left\|F_{l}\right\|^{2} \leq C_{9}(\phi) e^{3 C_{9}(\phi)}<\infty
\end{aligned}
$$

for almost all $\phi \in[0,2 \pi)$. Now, we apply Lemma 4 to system (4.2), putting $K_{l}=$ $e^{i \pi \frac{E}{F} \sigma_{3}} S_{l}$. As a result, we arrive at the required statement.

Proof of Theorem 2. Theorem 9 implies that system (5) has no subordinate solutions for almost any $E \in \mathbb{R}$ with respect to Lebesgue measure. Now, Theorem 2 is a consequence of Lemma 1.

\section{$\S 5$. Application}

This section is devoted to the proof of the fact that the operator

with domain

$$
H=-\frac{d^{2}}{d x^{2}}-F x+p(x) \quad \text { in } L_{2}\left(\mathbb{R}_{+}\right)
$$

$$
\operatorname{dom}(H)=\left\{\varphi: \begin{array}{l}
\varphi^{\prime} \text { is absolutely continuous, } \varphi(0)=0, \operatorname{supp}(\varphi) \text { is } \\
\text { bounded, and }-\varphi^{\prime \prime}+p \varphi \in L_{2}\left(\mathbb{R}_{+}\right)
\end{array}\right\}
$$

is essentially selfadjoint. The fact that the domain $\operatorname{dom}(H)$ is dense in $L_{2}\left(\mathbb{R}_{+}\right)$follows from [K] Chapter VI, $\S 4$, Theorem 4.2]. It can easily be checked that the operator $H$ is symmetric. Therefore, to prove that $H$ is essentially selfadjoint, it suffices to verify that $H^{*}$ is symmetric.

We introduce the operator $h=-\frac{d^{2}}{d x^{2}}-F x+p(x)$ with domain $\operatorname{dom}(h)=\{\varphi$ : $\varphi^{\prime}$ is absolutely continuous $\}$ and with values in $L_{1, \text { loc }}\left(\mathbb{R}_{+}\right)$.

Lemma 16. Let $v \in \operatorname{dom}\left(H^{*}\right)$. Then $v^{\prime}$ is absolutely continuous, $h v \in L_{2}\left(\mathbb{R}_{+}\right)$, and $v(0)=0$.

Proof. The definition of $H^{*}$ implies that for some $w \in L_{2}\left(\mathbb{R}_{+}\right)$we have

$$
\int_{0}^{\infty} h u(x) \bar{v}(x) d x=\int_{0}^{\infty} u(x) \bar{w}(x) d x
$$

for all $u \in \operatorname{dom}(H)$. Consider the equation $h \psi=w$ with initial conditions $\psi(0)=0$ and $\psi^{\prime}(0)=0$. Reducing this equation to an integral one and applying Picard's method, it is not hard to show that it is uniquely solvable. Moreover, from the integral representation it follows that $\psi^{\prime}$ is absolutely continuous. We note that, in general, the function $\psi$ does not belong to $L_{2}\left(\mathbb{R}_{+}\right)$. 
Let $\operatorname{supp}(u) \subset[0, l]$. Using $(5.1)$, we obtain

$$
\begin{aligned}
& \int_{0}^{\infty} h u(x) \bar{v}(x) d x=\int_{0}^{l} u(x) h \bar{\psi}(x) d x \\
& \quad=\int_{0}^{l} u(x)\left(-\bar{\psi}^{\prime \prime}(x)-F x \bar{\psi}(x)+p(x) \bar{\psi}(x)\right) d x \\
& \quad=-u(0) \bar{\psi}^{\prime}(0)+u^{\prime}(0) \bar{\psi}(0)+\int_{0}^{l} h u(x) \bar{\psi}(x) d x=\int_{0}^{\infty} h u(x) \bar{\psi}(x) d x .
\end{aligned}
$$

Thus, for all $u \in \operatorname{dom}(H)$ we have

$$
\int_{0}^{\infty} h u(x)(\bar{v}(x)-\bar{\psi}(x)) d x=0 .
$$

The operator $H_{a l}$ in $L_{2}[0, l]$ given by the formula $H_{a l} \varphi=h \varphi$ on the domain

$$
\operatorname{dom}\left(H_{a l}\right)=\left\{\begin{array}{l}
\varphi \varphi^{\prime} \text { is absolutely continuous, and } h \varphi \in \\
L_{2}[0, l], \varphi(0)=\varphi^{\prime}(l)=0
\end{array}\right\}
$$

is selfadjoint (see $\mathrm{K}$, Chapter VI, $\S 4$, Theorem 4.2]). Therefore, the kernel of $H_{a l}^{*}-i$ is zero. Consequently, there exists $\psi_{a} \in \operatorname{dom}(h)$ such that $h \psi=i \psi, \psi_{a}(0)=0$, and $\psi_{a}^{\prime}(l)=1$.

We consider the family of operators $H_{l}$ in $L_{2}[0, l]$ with domain

$$
\operatorname{dom}\left(H_{l}\right)=\left\{\varphi: \begin{array}{l}
\varphi^{\prime} \text { is absolutely continuous, } h \varphi \in L_{2}[0, l], \\
\text { and } \varphi(0)=\varphi(l)=\varphi^{\prime}(l)=0
\end{array}\right\}
$$

that are given by the rule $H_{l} \varphi=h \varphi$ for $\varphi \in \operatorname{dom}\left(H_{l}\right)$. It is clear that $\operatorname{dom}\left(H_{a l}\right)=$ $\operatorname{dom}\left(H_{l}\right) \dot{+}\left\{C \psi_{a}\right\}$. Since $H_{l} \subset H_{a l}=H_{a l}^{*} \subset H_{l}^{*}$, we see that the operator $H_{l}$ is closed and that the kernel of $H_{l}^{*}-i$ is one-dimensional. Moreover, it is easy to show that $\psi_{a} \in \operatorname{ker}\left(H_{l}^{*}-i\right)$, whence $\operatorname{ker}\left(H_{l}^{*}-i\right)=\left\{C \psi_{a}\right\}$. The domain of the adjoint operator looks like this:

$$
\operatorname{dom}\left(H_{l}^{*}\right)=\operatorname{dom}\left(H_{l}\right) \dot{+} \operatorname{ker}\left(H_{l}^{*}-i\right) \dot{+} \operatorname{ker}\left(H_{l}^{*}+i\right)=\operatorname{dom}\left(H_{l}\right) \dot{+}\left\{C \psi_{a}\right\} \dot{+}\left\{C \bar{\psi}_{a}\right\}
$$

(see $[\mathrm{BS}])$. Consequently,

$$
\operatorname{dom}\left(H_{l}^{*}\right) \subset\left\{\varphi: \varphi^{\prime} \text { is absolutely continuous, } h \varphi \in L_{2}[0, l]\right\} \equiv \Omega_{l} .
$$

Identity (5.2) is valid for the functions $u$ in $\operatorname{dom}\left(H_{l}\right)$ extended by zero to the region $x \geq l$. Therefore, $v-\psi \in \operatorname{dom}\left(H_{l}^{*}\right) \subset \Omega_{l}$. It follows that $v \in \Omega_{l}$. Since this is true for all $l>0, v^{\prime}$ is absolutely continuous on the semiaxis.

Since $v \in \Omega_{l}$, from (5.1) we deduce that

$$
\int_{0}^{\infty} u(x)(h \bar{v}(x)-\bar{w}(x)) d x=u^{\prime}(0) \bar{v}(0), \quad u \in \operatorname{dom}(H) .
$$

Consequently, $h v(x)=w(x) \in L_{2}[0, \infty)$ and $v(0)=0$.

Lemma 17. For every $\varepsilon>0$, there exists $N>0$ such that $p(x)=p_{\varepsilon}(x)+p_{0}(x)$,

$$
\int_{0}^{1}\left|p_{\varepsilon}(x)\right| d x \leq \varepsilon, \quad\left|p_{0}\right| \leq N .
$$

Proof. This follows from the fact that $p(x)$ is absolutely integrable.

Lemma 18. If $u$ is a continuously differentiable function, then

$$
\int_{0}^{\zeta}\left|p_{\varepsilon}(x)\right||u(x)|^{2} d x \leq 4 \varepsilon \int_{0}^{\zeta}\left|u^{\prime}(x)\right|^{2}+|u(x)|^{2} d x, \quad \zeta>0 .
$$


Proof. For each $\alpha \in[1,2]$, we have

$$
\begin{aligned}
& \int_{n}^{n+\alpha}\left|p_{\varepsilon}(x)\right||u(x)|^{2} d x \\
& \quad \leq \max _{n \leq x \leq n+\alpha}|u(x)|^{2} \int_{n}^{n+\alpha}\left|p_{\varepsilon}(x)\right| d x \leq 2 \varepsilon \max _{n \leq x \leq n+\alpha}|u(x)|^{2} \\
& \quad \leq 4 \varepsilon \int_{n}^{n+\alpha}\left|u^{\prime}(x)\right|^{2}+|u(x)|^{2} d x
\end{aligned}
$$

(the latter estimate is based on the fact that the embedding $H^{1}[0,1] \subset C[0,1]$ is continuous). Next,

$$
\begin{gathered}
\int_{0}^{\zeta}\left|p_{\varepsilon}(x)\right||u(x)|^{2} d x=\sum_{n=0}^{[\zeta]-2} \int_{n}^{n+1}\left|p_{\varepsilon}(x)\right||u(x)|^{2} d x+\int_{[\zeta]-1}^{\zeta}\left|p_{\varepsilon}(x)\right||u(x)|^{2} d x \\
\leq \sum_{n=0}^{[\zeta]-2} 4 \varepsilon \int_{n}^{n+1}\left|u^{\prime}(x)\right|^{2}+|u(x)|^{2} d x+4 \varepsilon \int_{[\zeta]-1}^{\zeta}\left|u^{\prime}(x)\right|^{2}+|u(x)|^{2} d x \\
=4 \varepsilon \int_{0}^{\zeta}\left|u^{\prime}(x)\right|^{2}+|u(x)|^{2} d x .
\end{gathered}
$$

Lemma 19. Let $u \in \operatorname{dom}\left(H^{*}\right)$. Then for all but finitely many $n \in \mathbb{N}$ there exists $\zeta_{n} \in[n, n+1)$ such that $\left|\operatorname{Re}\left(\bar{u}\left(\zeta_{n}\right) u^{\prime}\left(\zeta_{n}\right)\right)\right| \leq 1$.

Proof. Assume the contrary. Then for infinitely many intervals we have

$$
\left|\operatorname{Re}\left(u(x) \bar{u}^{\prime}(x)\right)\right|>1, \quad x \in[n, n+1) .
$$

For definiteness, assume that $\operatorname{Re}\left(u(x) \bar{u}^{\prime}(x)\right)>1$ (the case of $\operatorname{Re}\left(u(x) \bar{u}^{\prime}(x)\right)<-1$ is analyzed similarly). Then

$$
\begin{gathered}
|u(y)|^{2}-|u(n)|^{2}=\int_{n}^{y}\left(|u(x)|^{2}\right)^{\prime} d x=\int_{n}^{y} u(x) \bar{u}^{\prime}(x)+\bar{u}(x) u^{\prime}(x) d x \\
=\int_{n}^{y} 2 \operatorname{Re}\left(\bar{u}(x) u^{\prime}(x)\right) d x \geq 2(y-n),
\end{gathered}
$$

whence

$$
|u(y)|^{2} \geq 2(y-n), \quad \int_{n}^{n+1}|u(y)|^{2} d y \geq 1 .
$$

Since $u \in L_{2}\left(\mathbb{R}_{+}\right)$, the latter inequality cannot be true for infinitely many $n$.

Lemma 20. If $u \in \operatorname{dom}\left(H^{*}\right)$, then

$$
\int_{0}^{R}\left|u^{\prime}(x)\right|^{2} d x \leq A(u) R+B(u) .
$$

Proof. By Lemma 16, we have

$$
\begin{aligned}
\int_{0}^{\zeta_{n}} & \bar{u}(x) h u(x) d x=\int_{0}^{\zeta_{n}} \bar{u}(x)\left(-u^{\prime \prime}(x)-F x u(x)+p(x) u(x)\right) d x \\
& =\int_{0}^{\zeta_{n}}\left|u^{\prime}(x)\right|^{2} d x+\int_{0}^{\zeta_{n}} p(x)|u(x)|^{2} d x-\int_{0}^{\zeta_{n}} F x|u(x)|^{2} d x-\bar{u}\left(\zeta_{n}\right) u^{\prime}\left(\zeta_{n}\right) .
\end{aligned}
$$


Let $\|u\|$ denote the norm of $u$ in $L_{2}\left(\mathbb{R}_{+}\right)$. By Lemmas $16-19$, we have the estimate

$$
\begin{aligned}
\int_{0}^{\zeta_{n}} & \left|u^{\prime}(x)\right|^{2} d x \\
\leq & \int_{0}^{\zeta_{n}} F x|u(x)|^{2} d x+\int_{0}^{\zeta_{n}}|p(x)||u(x)|^{2} d x+\operatorname{Re}\left(\bar{u}\left(\zeta_{n}\right) u^{\prime}\left(\zeta_{n}\right)\right) \\
& +\int_{0}^{\zeta_{n}}|u(x)||h u(x)| d x \\
\leq & F \zeta_{n}\|u\|^{2}+\int_{0}^{\zeta_{n}}|p(x) \| u(x)|^{2} d x+1+C \\
\leq & F \zeta_{n}\|u\|^{2}+1+C+\int_{0}^{\zeta_{n}}\left|p _ { \varepsilon } ( x ) \left\|\left.u(x)\right|^{2} d x+\int_{0}^{\zeta_{n}}\left|p_{0}(x) \| u(x)\right|^{2} d x\right.\right. \\
\leq & \left(F \zeta_{n}+N\right)\|u\|^{2}+1+C+4 \varepsilon \int_{0}^{\zeta_{n}}\left|u^{\prime}(x)\right|^{2}+|u(x)|^{2} d x .
\end{aligned}
$$

Choosing $\varepsilon=1 / 8$, we obtain

$$
\int_{0}^{\zeta_{n}}\left|u^{\prime}(x)\right|^{2} d x \leq\left(2 F \zeta_{n}+2 N+1\right)\|u\|^{2}+2+2 C
$$

and the claim follows.

Lemma 21. The operator $H^{*}$ is symmetric.

Proof. Let $u, v \in \operatorname{dom}\left(H^{*}\right)$. Then

$$
\int_{0}^{R} h u \bar{v} d x-\int_{0}^{R} u h \bar{v} d x=-u^{\prime}(R) \bar{v}(R)+u(R) \bar{v}^{\prime}(R) .
$$

By Lemma 16, the left-hand side has a finite limit as $R \rightarrow \infty$; we denote this limit by $C_{u v}$. To prove the lemma, it suffices to show that $C_{u v}=0$. Using Lemma 20, we can write

$$
\int_{0}^{R}\left(-u^{\prime}(x) \bar{v}(x)+u(x) \bar{v}^{\prime}(x)\right) d x=C_{u v} R+o(R)
$$

and

$$
\begin{aligned}
& \left|\int_{0}^{R}\left(-u^{\prime}(x) \bar{v}(x)+u(x) \bar{v}^{\prime}(x)\right) d x\right| \leq \int_{0}^{R}\left|u^{\prime}(x)\|v(x)|+| u(x)\| v^{\prime}(x)\right| d x \\
& \quad \leq\left(\int_{0}^{R}\left|u^{\prime}(x)\right|^{2} d x\right)^{1 / 2}\|v\|+\left(\int_{0}^{R}\left|v^{\prime}(x)\right|^{2} d x\right)^{1 / 2}\|u\| \\
& \quad \leq\|v\| \sqrt{A_{u} R+B_{u}}+\|u\| \sqrt{A_{v} R+B_{v}} \leq A_{u v} \sqrt{R}+B_{u v}
\end{aligned}
$$

for some $A_{u v}>0$ and $B_{u v}>0$. Comparing the latter inequality with (5.4), we see that $C_{u v}=0$. The lemma is proved.

\section{REFERENCES}

[AGZ] J. Avron, L. Gunter, and J. Zak, Energy uncertainty in "Stark ladder", Solid State Comm. 16 (1975), no. 2, 189-191.

[AZ] J. Avron and J. Zak, Instability of the continuous spectrum: the N-band Stark ladder, J. Math. Phys. 18 (1977), no. 5, 918-921. MR0438948 (55:11851)

[B1] V. S. Buslaev, Kronig-Penney electron in a homogeneous electric field, Differential Operators and Spectral Theory, Amer. Math. Soc. Transl. (2), vol. 189, Amer. Math. Soc., Providence, RI, 1999, pp. 45-57. MR1730502 (2001b:81039) 
[DK] P. Deift and R. Killip, On the absolutely continuous spectrum of one-dimensional Schrödinger operators with square summable potentials, Comm. Math. Phys. 203 (1999), no. 2, 341-347. MR 1697600 (2000c:34223)

[DSS] F. Delyon, B. Simon, and B. Souillard, From power pure point to continuous spectrum in disordered systems, Ann. Inst. H. Poincaré Phys. Théor. 42 (1985), no. 3, 283-309. MR0797277 (87d:35098)

[E] P. Exner, The absence of the absolutely continuous spectrum for $\delta^{\prime}$ Wannier-Stark ladders, J. Math. Phys. 36 (1995), no. 9, 4561-4570. MR1347099 (96g:81050)

[GP] D. J. Gilbert and D. B. Pearson, On subordinacy and analysis of the spectrum of one-dimensional Schrödinger operators, J. Math. Anal. Appl. 128 (1987), no. 1, 30-56. MR0915965 (89a:34033)

[K] T. Kato, Perturbation theory for linear operators, Springer-Verlag, Berlin, 1995. MR1335452 (96a:47025)

[LS] Y. Last and B. Simon, Eigenfunctions, transfer matrices, and absolutely continuous spectrum of one-dimensional Schrödinger operators, Invent. Math. 135 (1999), no. 2, 329-367. MR1666767 (2000f:47060)

[NN] A. Nenciu and G. Nenciu, Dynamics of Bloch electrons in external electric fields. I. Bounds for interband transitions and effective Wannier Hamiltonians, J. Phys. A 14 (1981), no. 10, 2817-2827. MR0629327 (82j:81069)

[BS] M. Sh. Birman and M. Z. Solomyak, Spectral theory of selfadjoint operators in Hilbert space, Leningrad. Gos. Univ., Leningrad, 1980; English transl., D. Reidel Publishing Co., Dordrecht, 1987. MR0609148 (82k:47001) MR1192782 (93g:47001)

[B2] V. S. Buslaev, Adiabatic perturbation of a periodic potential, Teoret. Mat. Fiz. 58 (1984), no. 2, 233-243; English transl., Theoret. and Math. Phys. 58 (1984), no. 2, 153-159. MR0743409 (85i:34032)

[Ba] M. V. Buslaeva, The one-dimensional Schrödinger operator with accelerating potential, Funktsional. Anal. i Prilozhen. 18 (1984), no. 1, 65-66; English transl., Functional Anal. Appl. 18 (1984), no. 1, 53-55. MR0739094 (86f:81026)

[BD1] V. S. Buslaev and L. A. Dmitrieva, Adiabatic perturbation of a periodic potential. II, Teoret. Mat. Fiz. 73 (1987), no. 3, 430-442; English transl., Theoret. and Math. Phys. 73 (1987), no. 3, 1320-1329. MR0939788 (89c:34058)

[BD2] — A Aloch electron in an external field, Algebra i Analiz 1 (1989), no. 2, 1-29; English transl., Leningrad Math. J. 1 (1990), no. 2, 287-320. MR1025153 (91g:81162)

[BF] V. S. Buslaev and L. D. Faddeev, Formulas for traces for a singular Sturm-Liouville differential operator, Dokl. Akad. Nauk SSSR 132 (1960), no. 1, 13-16; English transl., Soviet Math. Dokl. 1 (1960), 451-454. MR0120417 (22:11171)

[Z] A. Zygmund, Trigonometric series, Cambridge Univ. Press, Cambridge, 1988. MR0933759 (89c:42001)

[L] B. M. Levitan, Inverse Sturm-Liouville problems, "Nauka", Moscow, 1984; English transl., VSP, Zeist, 1987. MR0771843 (86d:34002); MR0933088 (89b:34001)

[P] A. A. Pozharski1̌, Wannier-Stark type operators with singular potentials, Algebra i Analiz 14 (2002), no. 1, 158-193; English transl., St. Petersburg Math. J. 14 (2003), no. 1, 119-145. MR 1893324 (2003e:81051)

Department of Physics, St. Petersburg State University, Ulyanovskaya 1, Petrodvorets, St. Petersburg 198504, Russia

E-mail address: lehman@sbor.net

Received 10/AUG/2003

Translated by B. M. BEKKER 